\title{
Heterozygote advantage for the phenylketonuria allele
}

\author{
LETTEN FEGERSTEN SAUGSTAD \\ Vestheimgt. 6, Oslo 2, Norway
}

SUMMARY Mean weight at birth of unaffected (normal homozygous and PKU heterozygous) offspring of parents heterozygous for the phenylketonuria (PKU) allele averages significantly above that of Norwegian neonates, with no significant difference in mean age of mothers or in mean parity. It approaches the optimal birthweight - that which confers the minimum overall mortality in the pre-, peri-, and postnatal periods. This near-optimal birthweight together with the possibly higher effective fertility observed in PKU heterozygous couples (at least in those who married before 1940), has apparently more than outweighed the disadvantages of the allelele in PKU homozygous offspring as shown, for example, in an excessive number of pre- and perinatal deaths among the total offspring of PKU heterozygotes, to say nothing of the PKU survivors who, often, used to die young. The two effects-fertility and viability - apparently both contribute in the same direction, to give a biological fitness in excess of 1 for the heterozygote. This heterozygote advantage presumably explains the presence of the allele at frequencies above those to be expected from the simple replacement of a homozygously-lethal allele by mutation alone.

The prevalence at birth of recessive disorders is affected inter alia by changes in inbreeding level and by any selective advantage in the heterozygous carrier. Heterozygote advantage has been observed in man in sickle-cell anaemia and possibly in some other disorders (see Motulsky, 1974; Stuart and Burdon, 1974).

Evidence is presented for the view that heterozygosity for the phenylketonuria (PKU) allele is advantageous in parents by rendering their unaffected offspring more viable at birth, thereby improving the chance of survival of the allele.

\section{Material and methods}

The mortality rate (incorporating the stillbirth rate) for infants before 1 month of age is lowest among those with birthweights at a point somewhat above the mean (Karn and Penrose, 1951; Fraccaro, 1955; Rantakallio, 1968). This is also the case in regard to all births in Norway 1967-1968 (Bjerkedal and Bakketeig, 1972). There, the rate is lowest for birthweights in the neighbourhood of $4500 \mathrm{~g}$ (males), or $4000 \mathrm{~g}$ (females): while the average birthweight was $3512 \mathrm{~g}$ for males and $3391 \mathrm{~g}$ for females (Table 1). Received for publication 12 March 1976
The chances for further survival to 1 year of age appeared to be best for neonates of either sex that had a weight at birth of approximately $4500 \mathrm{~g}$.

Weights at birth of children with PKU born in Norway between 1940 and 1973 and of their nonPKU sibs were investigated, using the information in the maternity hospitals (Saugstad, 1972). Controls were all births in Norway between January 1967 and January 1969 from the Medical Registration of Births (T. Bjerkedal, 1973, personal communication), which is based on forms completed on the ninth day of life.

The number of pregnancies (including abortions and pre- and perinatal deaths) of the heterozygous mothers with PKU offspring were also investigated and compared with a control series of 230 nonprimiparous women, of unknown heterozygosity for PKU, consecutively admitted for delivery in Oslo in 1967. In addition, the numbers of liveborn offspring in 36 families with PKU offspring born in Norway before 1940 were investigated (Saugstad, 1975a). The late Asbjorn Følling kindly supplied information on 22 of the 36 families diagnosed by him in 1932 to 1938 inclusive (Følling et al., 1944). The remaining 14 of the 36 families were investigated by the present author 1968 to 1971, when the reproductive histories 
Table 1 Mean birthweight, mean duration of pregnancy, and rate of pregnancy abnormalities in 86 non-PKU offspring of PKU heterozygous women and in all births in Norway 1967 and 1968

\begin{tabular}{|c|c|c|c|c|c|c|c|}
\hline & \multicolumn{3}{|c|}{ Mean birthweight (g) } & \multicolumn{4}{|l|}{ Pregnancy } \\
\hline & Male & Femalc & Total & Duration & Toxaemia & Bleeding & $\begin{array}{l}\text { Preterm } \\
\text { births }\end{array}$ \\
\hline $\begin{array}{l}86 \text { Non-PKU offspring } \\
135695 \text { Controls }\end{array}$ & $\begin{array}{l}3790 \text { (SD 519) } \\
3512 \text { (SD 612) }\end{array}$ & $\begin{array}{l}3778 \text { (SD 400) } \\
3391 \text { (SD 591) }\end{array}$ & $\begin{array}{l}3785 \text { (SD 465) } \\
3451 \text { (SD 615) }\end{array}$ & $\begin{array}{l}273(d) \\
278\end{array}$ & $\begin{array}{l}19.5 \% \\
5.0 \%\end{array}$ & $\begin{array}{r}20 \cdot 4 \% \\
2.4 \%\end{array}$ & $\begin{array}{l}20.1 \% \\
10.4 \%\end{array}$ \\
\hline Differences & 278 (SE 75) & 387 (SE 65) & 332 (SE 52) & & & & \\
\hline
\end{tabular}

Toxaemia has been defined as blood-pressure above $140 / 95 \mathrm{mmHg}$, plus proteinuria and oedema. Pr eterm births $=37$ weeks or less.

Mean parity of the 135695 controls was $2 \cdot 24$, with $36 \cdot 9 \%$ first-borns, $29 \cdot 8 \%$ second-borns, $18 \cdot 3 \%$ third-borns, $9 \cdot 1 \%$ fourth-borns. There were $6.1 \%$ of higher parity, with a mean parity of 6.1 . Mean age of control mothers $=26.74$ years, and of PKU heterozygous mothers when delivering the non-PKU offspring $=26 \cdot 54$ years.

In the controls, mean birthweight of both sexes combined increased by $140 \mathrm{~g}$ from first- to second-born, and by $55 \mathrm{~g}$ between second- and laterborns (Saugstad, 1972). Mean birthweight for the controls was maximal for the fourth child (males) and the fifth child (females) and thereafter slightly declined. The sex difference in mean birthweight ranged from $117 \mathrm{~g}$ (in first-borns) to $161 \mathrm{~g}$ (in fourth-borns), with a mean of $140 \mathrm{~g}$.

were obtained from the various obstetric departments.

\section{Birthweights}

Comparisons of PKU children with their sibs, as regards birthweight, were not possible in 36 of the 1940-1972 set of 81 families for the following reasons: in 24 families the PKU child was the only living child; in 5 of the families with 2 children each, both children were affected; in 7 families, information was incomplete.

In the remaining 43 families-those with both PKU and non-PKU offspring - the birthweights could be compared. Those of non-PKU offspring averaged significantly above those of the PKUs, in spite of the mean duration of pregnancy being 10 days shorter, and in spite of there being no difference in the high frequency of pregnancy abnormalities (Saugstad, 1972) (Table 2). Mean parity was 2.25 for the non-PKU offspring as compared with $2 \cdot 88$ for the PKUs.

The estimated correlation coefficient between birthweights of affected sibs and those of unaffected (non-PKU) sibs was +0.35 (103 pairs), whereas the correlation coefficient between unaffected sib and unaffected sib with reference to birthweight was approximately +0.54 (68 pairs). The mean birthweight of the non-PKU offspring also averaged significantly above Norwegian neonates born between January 1967 and January 1969, despite a shorter mean duration of pregnancy, and despite there being no particular difference in mean parity or in mean age of mothers (Table 1). There was only a negligible difference in birthweight between the sexes among the non-PKU offspring, as compared with a male excess of $121 \mathrm{~g}$ among all Norwegian neonates. The mean birthweight of the non-PKU offspring showed only a negligible increase with parity (Saugstad, 1972), whereas mean birthweight of the controls increased by about $140 \mathrm{~g}$ from the first to the second
Table 2 Mean weight at birth for 49 children with phenylketonuria and for their 86 sibs

\begin{tabular}{llll}
\hline \multicolumn{4}{l}{ Mean birthweight $(\mathrm{g})$} \\
\cline { 2 - 4 } & Male & Female & Total \\
\hline 86 Non-PKU sibs & 3790 SD 519 & 3778 SD 400 & 3785 SD 465 \\
49 PKU sibs & $(48)$ & $(38)$ & $(86)$ \\
& $(23)$ & $(26)$ & $(49)$ \\
\hline Differences & 188 SE 167 & 503 SE 141 & 356 SE 109 \\
\hline
\end{tabular}

Number of cases in brackets.

Within the 43 sibships, the PKU sib averaged $15.0 \%$ below the mean birthweight of the non-PKU sibs. The standard deviations of the PKU and non-PKU groups were, respectively, $14.4 \%$ and $5.5 \%$ of the mean birthweight of the non-PKU sibs.

The percentages of children by increasing parity are, respectively,

$26 \cdot 5,28 \cdot 6,8 \cdot 2,22 \cdot 4,14 \cdot 3,4 \cdot 1,6 \cdot 2,2 \cdot 0,0,2.0$ among the PKU

children (mean parity $2 \cdot 88$ ) and
$32 \cdot 6,31 \cdot 4,26.7,3 \cdot 5,2 \cdot 3,0,3 \cdot 5,0,0,0$ among the nonPKU sibs (mean parity $2 \cdot 25$ ).

child, and thereafter only slowly until after the fourth child (T. Bjerkedal, 1973, personal communication). This is in agreement with observations by other authors (James, 1969).

\section{Fertility}

Family size is sometimes limited after the birth of a child with a severe handicap, particularly if it is known to be a genetic disorder. If the diagnosis of PKU in one of the offspring was made before the introduction of dietary treatment, one would, therefore, expect a reduced mean number of children in families with affected offspring, as compared with the general population. In 4 of the 8 families that had 2 children each, both children were affected and the parents were advised to and did limit family size (A. Følling, 1972, personal communication). The PKU child was the lastborn in the remaining families with 2 children, in all families with 3 or 4 children, and in all except 1 of the 4 families with 5 living children (Saugstad, 1975a). 
Table 3117 families with $156 P K U, 191$ non-PKU, and 20 deceased offspring classified by size of sibship and by parents' year of marriage

\begin{tabular}{lll}
\hline Size of sibship & \multicolumn{2}{l}{ Year of marriage } \\
\cline { 2 - 3 } & Before 1940 & After 1940 \\
\cline { 2 - 3 } & Completed families & $\begin{array}{l}\text { Including some } \\
\text { incomplete families }\end{array}$ \\
\hline 1 & 0 & 22 \\
2 & $8^{*}$ & 31 \\
3 & $6 \dagger$ & 18 \\
4 & $2 \dagger$ & 8 \\
5 & 4 & 1 \\
6 & 6 & 1 \\
7 & 3 & 0 \\
8 & 0 & 0 \\
9 & 4 & 0 \\
10 & 2 & 0 \\
11 & 1 & 0 \\
\hline Total number of & 36 & 81 \\
families & & $2 \cdot 2$ \\
\hline Mean number of & & \\
children per family & $5 \cdot 2$ & \\
\hline
\end{tabular}

In Norway as a whole, the mean sibship size (excluding zero size) was, respectively, $3.45,2 \cdot 40,2.28$ in the years 1920 to $1945,1950,1960$ (G. S. Lettenstrøm, 1974, personal communication).

*In 4 of the 8 of the pre-1940 group that had 2 children, both children were affected, and the PKU child was the last-born in the remaining 4 families.

†The PKU child was the last-born child in all families with 3 or 4 children.

But, despite limitation, of the 36 couples with PKU offspring who married before 1940, the mean sibship size was $5 \cdot 2$ children as compared with 3.45 for non-zero sibships in the general population 1920 to 1945 inclusive (Table 3). Induced abortions had been performed in these families, so the mean of 5.2 children does not therefore reflect the total fertility of the 36 PKU heterozygous couples.

When the parents married after January 1940 (i.e. 1940 to 1970 inclusive), the mean number of liveborn children born into the 81 affected sibships averaged $2 \cdot 2$, slightly below that for the general population at that time (about 2.4 per sibship). On the other hand, a comparison between non-primiparous PKU heterozygous women who delivered in 1941 to 1972 inclusive and a control series of non-primiparous mothers of unknown but infrequent heterozygosity for PKU, who delivered in 1967 (Table 4), reveals 3.15 liveborn offspring per PKU heterozygous woman as compared with $2 \cdot 10$ children in the control-series. There was no particular difference in mean age at the start of childbearing in the two groups.

The mean year of delivery of PKU heterozygotes was 1959. That for controls was 1964 . The mean number of pregnancies per PKU heterozygote was 4.09 (cf. 2.34 for controls) and the discrepancy between the mean number of pregnancies and the number of liveborn children is the result of pre- and perinatal deaths and of spontaneous abortions
Table 4 Reproductive competence among 45 nonprimparous $P K U$ heterozygous mothers delivered 1941-1972 inclusive, and 230 non-primiparous mothers of unknown heterozygosity for $P K U$, consecutively admitted for delivery in Oslo City Hospital in 1967

\begin{tabular}{lcc}
\hline & $\begin{array}{l}\text { PKU } \\
\text { heterozygotes }\end{array}$ & Controls \\
\hline Livebirths & 142 & 485 \\
Stillbirths and perinatal deaths & 8 & 7 \\
Spontaneous abortions & 37 & 47 \\
Total number of pregnancies & $184^{*}$ & 539 \\
Number of pregnancies per mother & $4 \cdot 09$ & $2 \cdot 34$ \\
Number of liveborn children per mother & $3 \cdot 15$ & $2 \cdot 10$ \\
\hline
\end{tabular}

Mean age of PKU heterozygous mothers at delivery of first child was 23.74 years (cf. 23.80 years for controls). Five of them started childbearing in 1940 to 1950,20 in 1950 to 1960 , and the remaining 20 in 1960 to 1970 . Mean year of delivery 1959 (1964 for the control series). All had both PKU and non-PKU offspring. Reproductive histories were obtained from hospital records. Of the control series consisting of 330 mothers consecutively admitted for delivery in Oslo City Hospital in 1967, 98 mothers were primiparous and have been excluded. Two others were excluded-a sister of a known PKU homozygote, and a mother who delivered a PKU homozygote in 1972.

*There were 3 twin pregnancies, and there was a stillbirth in each of 2 of these.

(Saugstad, 1973). As regards the control series, the rates of prenatal and perinatal deaths and abortions are fairly well in accordance with those in the general population in 1967.

\section{Discussion}

The mean weight at birth of non-PKU offspring of PKU heterozygous women averages significantly above that of Norwegian neonates.

The question arises whether this increased mean weight at birth of non-PKU offspring could be related to an excess of later born children among the non-PKUs, as mean birthweight increases with parity. However, mean parity for the non-PKUs was 2.25 as compared with 2.24 for the Norwegian neonates delivered in 1967 and 1968. Of the 86 non-PKU offspring, only $8(9.3 \%)$ were of parity 4 or more, as compared with $15.3 \%$ of the controls. More particularly, the mean birthweight of non-PKUs increased negligibly with parity (I-II $=-27 \mathrm{~g}, 1-\mathrm{III}=+18 \mathrm{~g}$ ). There is a non-significant reduction of the usual male excess in birthweight (say $120 \mathrm{~g}$ ) in regard to the nonPKU offspring ( +12 g, S.E. 99).

Within the affected sibships, the variation in birthweight of the unaffected (non-PKU) offspring was small (SD 5.5\% of the sibship mean). An investigation of 47 well-to-do families in Oslo with 4 or more children, showed a standard deviation of $7.9 \%$ of the sibship mean. This is higher than the value of $4.6 \%$ obtained by Turner (1971) in his investigation of 55 
well-to-do families with 4 or more children. However, he adjusted the firstborn weight to that expected for a second-born, and in the 47 Norwegian families that adjustment (the mean difference in birthweight between the first two children) would have been $+9 \cdot 1 \%$, with a consequent reduction in standard deviation. The mean birthweight of the non-PKU offspring of PKU heterozygous women approaches the optimum birthweight-that which carries the lowest pre- and perinatal mortality rates-thereby improving the chances of survival of the PKU mutant allele. This heterozygote advantage-fast fetal growth of nonPKU offspring (i.e. normal homozygous and PKU heterozygous)-is perhaps sufficient to explain the observed survival of the allele at frequencies unlikely to be maintained by fresh mutation alone. In Norway there exists a seasonal variation in infant mortality with a minimum survival rate in January, February, and March, the coldest months of the year. The chance for further survival to 1 year of age appears also to be least for those infants born during these months (Bjerkedal and Bakketeig, 1972). For survival neonatally and in early infancy, a birthweight above the mean is certainly advantageous in cold climates, and it was probably of even greater importance in the past when mortality rates were higher.

In the 1830's in Norway, the overall mortality was $14 \%$ ranging from above $20 \%$ in the coastal deaneries to values below $10 \%$ in the inland deaneries (Drake, 1969; G. S. Lettenstrøm, 1974, personal communication).

If we accept the possible hypothesis that the PKU mutant allele was brought to Norway from the Western Viking settlements, 1000 or more years ago by immigrants (Saugstad, 1975b), the observed excess of growth potential in utero among the female carriers of the allele may have been a particularly important factor in the survival of the allele, from that time.

A birthweight that is greater in unaffected sibs than in the controls has also been observed in sibs of children with cystic fibrosis of the pancreas, where mean birthweight of unaffected male sibs was 142.6 (SE 73.6 g) greater, and that of unaffected female sibs $137.8 \mathrm{~g}$ (SE 64.3 g) greater than that of controls, whereas the difference in birthweight between affected and unaffected sibs was $245.5 \mathrm{~g}$ (SE 106.30 g) for males and $273.6 \mathrm{~g}$ (SE 100.26 g) for females (Hsia, 1959). The correlation of birthweight of unaffected with affected sib was low $(+0.35,181$ pairs), whereas the correlation of birthweight of unaffected with unaffected sib was +0.53 (89 pairs). An investigation of galactosaemia (Hsia and Walker, 1961) has also revealed a mean birthweight of unaffected sibs significantly above that of their affected sibs.
Of the PKU heterozygous women investigated, only one has developed diabetes, and glucose tolerance tests were unremarkable in 2 PKU heterozygotes with overweight firstborns (birthweight above $4500 \mathrm{~g}$ ). In PKU homozygous children, unremarkable glucose tolerance and insulin response tests have been recorded (Hefferman, 1971). The observation that few of the mothers of overweight infants develop diabetes, and that about $90 \%$ of the infants have unremarkable glucose disappearance rates, also makes it unlikely that hyperglycaemia is responsible for the fast fetal growth (Saugstad, 1972).

In regard to the observed possibly higher fertility among PKU heterozygous couples who married before 1940, it should be remembered that in the majority of the 36 couples, the diagnosis of PKU in the offspring was made before the end of the reproductive period, and several induced abortions have actually been performed. The observed mean of 5.2 children liveborn, therefore, underestimates the true fertility of these 36 PKU heterozygous couples.

Whereas it seems reasonable to conclude that the diagnosis of PKU in an offspring before dietary treatment tended to reduce (limit) family size, the indirect effect of dietary treatment on family size is more difficult to assess. The degree of limitation of family size may be related to success or failure of dietary treatment, to the number of previously-born unaffected children, and to a variety of personal, psychological, and economic factors. In sibships detected through PKU screening programmes 1965 to 1973 inclusive, the mean number of children amounts to 3.0 as compared with 2.28 in the general population according to the 1960 census.

It is difficult to know whether an observed frequency of spontaneous abortions is raised or not, as long as the true frequency of abortion is not known, nor how often an early abortion is really a delayed menstruation or vice versa.

A control series known to be non-heterozygous for PKU and matched with the PKU heterozygotes for age, parity, socioeconomic, and geographic conditions was not available. The choice of a control series of mothers delivering in 1967 was influenced by the introduction of the Medical Registration of Births in Norway in the same year, as this enabled a comparison to be made between the control series and Norwegian mothers delivering in 1967 (Saugstad, 1973). Information on reproductive incompetence was obtained from the hospital records in regard to the control series as well as in regard to the PKU heterozygous women. It is conceivable that reproductive compensation for the excessive number of abortions and pre- and perinatal deaths could 
account for the observed greater effective fertility (number of liveborn children) among the PKU heterozygous couples than in the control series. Though there is no simple relation between a tendency to spontaneous abortions and fertility, it has been seen that at least a proportion of abortion-prone women tend to do more than replace their loss (James, 1961, 1963). One might also consider the excess of spontaneous abortions and pre- and perinatal deaths among offspring of PKU heterozygous women to be an accurate reflection of the elimination of PKU homozygous fetuses. The discrepancies between the findings of Blumina (1972) and Woolf et al. (1975) in regard to a possibly increased reproductive incompetence among PKU heterozygous couples could be the result in part of any difference that might exist in the proportion of PKU homozygous fetuses, and/or in part because of a difference between the two series as regards fertility. Any such differences would presumably be a consequence of differences in selection procedures between the two series. As biochemical diagnosis cannot be made prenatally at so early a stage, the question cannot be settled. It seems unlikely that the great proportion of rhesus-negatives among the heterozygotes in the present series could account for their increased reproductive incompetence, since no particular excess of rhesus-negatives has been observed among abortionprone women, and since Blumina (1972) had excluded rhesus-negative women as well as primiparae from her series of 155 PKU heterozygotes.

Unaffected offspring of PKU heterozygous women have apparently a birthweight advantage leading to an increased viability at birth; thereby improving the chances for survival of the allele. Such an advantage together with the possibly higher effective fertility among PKU heterozygous couples as observed in the present series among couples with PKU offspring who married before 1940, has apparently more than outweighed the disadvantage of the allele in PKU homozygous offspring. Notwithstanding the apparently low variance of birthweight in the genetically heterogeneous group of 'unaffected' sibs, we do not yet know that the birthweight advantage applies to offspring of both genotypes. For the moment we can only state that it is one or the other or both that shows the higher birthweight compared with the controls.

\section{References}

Bjerkedal, T., and Bakketeig, L. (1972). Medical Registration of Births in Norway, 1967-1968, p. 144. Bergen University, Bergen, Norway.

Blumina, M. G. (1972). Role of women's heterozygosity for phenylketonuria in the origination of spontaneous abortions and damages of the fetus development. Genetika, 8, 132-138.

Drake, M. (1969). Population and Society in Norway 17351865, p. 94, Table 4-14. Cambridge University Press, London.

Følling, A., Mohr, O. L., and Ruud, L. (1944). Oligophrenia phenylpyrouvica: a recessive syndrome in man. Skrifter utgitt av det Norske Videnskaps-Akademi $i$ Oslo. I. Mat.Naturv. Klasse, 13, 3-48.

Fraccaro, M. (1955). A contribution to the study of birthweight based on an Italian sample. Annals of Human Genetics, 20, 282-297.

Hefferman, A. G. A. (1971). Glucose tolerance and insulin response in phenylketonuria. New England Journal of Medicine, 285, 57-58.

Hsia, D. Y-Y. (1959). Birthweight in cystic fibrosis of the pancreas. Annals of Human Genetics, 23, 289-299.

Hsia, D. Y-Y., and Walker, F. A. (1961). Variability in the clinical manifestations of galactosemia. Journal of Pediatrics, 59, 872-883.

James, W. H. (1961). On the possibility of segregation in the propensity to spontaneous abortion in the human female. Annals of Human Genetics, 25, 207-213.

James, W. H. (1963). Notes towards an epidemilogy of spontaneous abortions. American Journal of Human Genetics, 15, 223-240.

James, W. H. (1969). Birthweight and birth order. Annals of Human Genetics, 32, 411-412.

Karn, M. N., and Penrose, L. S. (1951). Birthweight and gestation time in relation to maternal age, parity and infant survival. Annals of Eugenics, 16, 147-163.

Motulsky, A. G. (1974). Significance of genetic diseases for population studies. Israel Journal of Medical Sciences, 9, 1410-1416.

Rantakallio, P. (1968). The optimum birthweight. Annales Paediatriae Fenniae, 14, 66-70.

Saugstad, L. F. (1972). Birthweights in children with phenylketonuria and in their siblings. Lancet, 1, 809-813.

Saugstad, L. F. (1973). Increased 'reproductive casualty' in heterozygotes for phenylketonuria. Clinical Genetics, 4, 105-114.

Saugstad, L. F. (1975a). Frequency of phenylketonuria in Norway. Clinical Genetics, 7, 40-51.

Saugstad, L. F. (1975b). Anthropological significance of phenylketonuria. Clinical Genetics, 7, 52-61.

Stuart, A. B., and Burdon, M. G. (1974). Frequency of cysticfibrosis gene. Lancet, $2,1521$.

Turner, G. (1971). Recognition of intra-uterine growth retardation by considering comparative birthweights. Lancet, 2, 1123-1124.

Woolf, L. I., McBean, M. S., Woolf, P. M., and Cahalane, S. F. (1975). Phenylketonuria as a balanced polymorphism: the nature of the heterozygote advantage. Annals of Human Genetics, 38, 461-469. 